\title{
SALICETUM ALBAE ISSLER 1924 LEUCOJETOSUM AESTIVI PÎNZARU SUBASS. NOV. IN THE REPUBLIC OF MOLDOVA
}

\author{
Pavel PÎNZARU $1,2,3$ \\ ${ }^{1}$ National Botanical Garden (Institute) “Alexandru Ciubotaru”, Chisinau - Republic of Moldova \\ ${ }^{2}$ State Agrarian University of Moldova \\ ${ }^{3}$ Tiraspol State University, Chisinau - Republic of Moldova \\ E-mail: p_panzaru@yahoo.it
}

\begin{abstract}
This article is focused on the description of the forests of Salix alba L. with Leucojum aestivum L., in the valley of Prut River, in the Republic of Moldova. Based on 19 relevés, the author has grouped these forests in a plant community newly described for science - Salicetum albae leucojetosum aestivi subass. nov. included in the alliance Salicion albae Soó 1951, the order Salicetalia purpureae Moor 1958, cl. SALICETEA PURPUREAE Moor 1958.
\end{abstract}

Key words: Salicetum albae Issler 1924 leucojetosum aestivi subass. nov. characteristic of phytocoenosis, ecology, range, Republic of Moldova.

\section{Introduction}

Salix alba L. riverside forests, in the Republic of Moldova, occur in patches in the valleys of the Dniester River and the Prut River, very rarely and along streams in the central part of the country. From 1960 to 1995 , willow groves were studied according to the dominant method and grouped into the following associations: Salicetum (albae) inundatum and Salicetum (albae) rubosum (caesii) [GHEIDEMAN \& al. 1964; PÎNZARU, 1991; POSTOLACHE, 1995].

Since the beginning of the 21st century, the methods of the Central European School [BRAUN-BLANQUET, 1964] have been used in the phytocoenological research on the floodplain forests in the Republic of Moldova. Thus, the Salix alba forests from the scientific reserves "Plaiul Fagului" [POSTOLACHE \& CHIRTOACĂ, 2005], "Prutul de Jos" [POSTOLACHE \& POSTOLACHE, 2012]; "Pădurea Domnească" [TOFAN-BURAC \& CHIFU, 2002; POSTOLACHE, 2017] and from the "Nemţeni" forest reserve [COVALI, 2008] have been grouped in the association Salicetum albae Issler 1924 (= Salicetum albae Issler 1926) [GAFTA \& al. 2008].

The association Salicetum albae Issler 1924 has been included in the priority habitats, EU code 91E0, cf. Annex 1 Habitats Directive 92/43/EEC.

This article contains the description of a new subassociation of Salix alba L. with Leucojum aestivum L. leucojetosum aestivi Pînzaru subass. nov. as part of the association Salicetum albae Issler 1924.

\section{Materials and methods}

The phytocoenological studies in the field were carried out in the spring and summer of 2018, in the floodplain of the Prut River, between Nemţeni commune, Hînceşti district, at the north and Sărata Răzeşi commune, Leova district, at the south. For the preparation of this paper, we examined the descriptions of the forest plots from "Amenajările silvice" ("Forest Planning"), 2011-2014, ICAS, Chisinau. Nineteen relevés were described according to the 
methods of the Central European School [BRAUN-BLANQUET, 1964]. The area of a relevé was $600 \mathrm{~m}^{2}$, according to the school of Cluj-Napoca [CRISTEA \& al. 2004]. The list of species is presented in accordance with recent publications [SÂRBU \& al. 2013; PÎNZARU $\&$ SÎRBU, 2016]. Air temperature and atmospheric precipitation - according to the Atlas of Climate Resources of the Republic of Moldova [NEDEALCOV \& al. 2013]. Information on phytocoenological relevés, published in the Republic of Moldova [TOFAN-BURAC \& CHIFU, 2002; POSTOLACHE \& CHIRTOACĂ, 2005; COVALI, 2008; POSTOLACHE \& POSTOLACHE, 2012; POSTOLACHE, 2017], Romania [CHIFU \& MITITELU, 1992; IVAN \& al. 1993; GAFTA \& al. 2008; CHIFU \& IRIMIA, 2014; COLDEA, 2015], Germany [SEIBERT \& CONARD, 1992]; Cehia [NEUHÄUSLOVÁ \& DOUDA, 2013] and Slovenia [SÍLC, 2003], was examined for comparison.

\section{Results and discussions}

The association Salicetum albae Issler 1924 includes the phytocoenoses of Salix alba L. from the floodplains of rivers and streams in the Central and South-Eastern Europe. The following species are characteristic of the given association: Salix alba L., Salix fragilis L., Populus alba L., Populus nigra L., Fraxinus angustifolia Vahl, Acer negundo L., Rubus caesius L., Urtica dioica L., Phalaris arundinacea L., Aristolochia clematitis L., Humulus lupulus L., Iris pseudacorus L., Symphytum officinale L., Calystegia sepium (L.) Br. [CHIFU \& MITITELU, 1992; SEIBERT \& CONARD, 1992; IVAN \& al. 1993; SÍLC, 2003; NEUHÄSLOVÁ \& DOUDA, 2013; COLDEA, 2015].

Willow forests usually include few species, ephemeral plants are very few or absent. Analysing the list of species of the relevés of the association Salicetum albae Issler 1924 [CHIFU \& MITITELU, 1992; SEIBERT \& CONARD, 1992; IVAN \& al. 1993; TOFAN-BURAC \& CHIFU, 2002; SÍLC, 2003; POSTOLACHE \& CHIRTOACĂ, 2005; COVALI, 2008; POSTOLACHE \& POSTOLACHE, 2012; CHIFU \& IRIMIA, 2014; COLDEA, 2015; POSTOLACHE, 2017], we didn't find any mention of the presence of summer snowflake (Leucojum aestivum L.), although it was cited as a characteristic species of the alliance Salicion albae Soó 1930 [SÂRBU \& al. 2013]. This mesohygrophylic, geophyte is a central-europeanmediterranean-atlantic geoelement, which occurs naturally in hilly areas of several countries and regions: from Spain to Ukraine, Turkey, the Caucasus and Iran. This species is considered characteristic of the coenotaxa Calthion palustris Tx. 1937 [ELLENBERG \& al. 1992] and Fraxinetalia Scamoni et Passarge 1959 [AESCHIMANN \& al. 2004].

In the Republic of Moldova, the presence of the species Leucojum aestivum L. has been identified only in the willow groves of the valley of Prut River, in the vicinity of Sărata Răzeşi commune, Leova district, and Cioara and Dancu communes, Hînceşti district. It is a critically endangered species (CR), included in the Red Book of the Republic of Moldova [GHENDOV \& CIOCÂRLAN, 2015)]. As a result of the phytosociological studies carried out recently, we have found new places where this species occurs - near the communes Nemţeni, Cotul Morii, Leuşeni (near the custom house) and Călmăuţi, Hînceşti district. It grows sporadically or in abundant clusters (AD 2-4) in Salix alba L. riverside forests. Under the climatic conditions of the Republic of Moldova, Leucojum aestivum plants grow up to 75 $\mathrm{cm}$ tall and produce 3-6 leaves, which grow about $75 \mathrm{~cm}$ long and 10-16 mm wide. It blooms in April-May, produces by (1) 3-7 (8) pendant flowers, located unilaterally on pedicels of different lengths. The fruits are fleshy, ovate-triangular capsules, 10-25 mm long, 7-17 mm wide, with (1) 3-8 seeds in a fruit. It releases the seeds at the end of June - beginning of July. The seeds are spheroidal, about $6 \mathrm{~mm}$ in diameter, blackish. It propagates by seeds and vegetatively (Figure 1). 


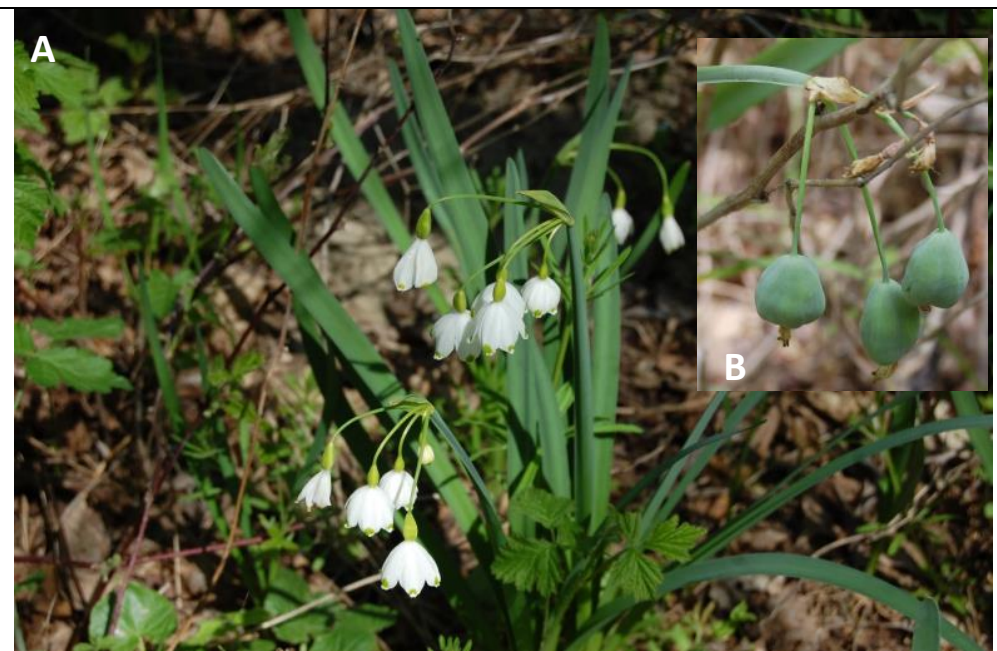

Figure 1. Leucojum aestivum L.: A. flowers - 21.IV.2018, B. fruits - 10.VI.2018

We group the plant communities of Salix alba L. with Leucojum aestivum L., accompanied by the constant species Ficaria verna Huds., Iris pseudacorus L. and Carex riparia Curtis in a new subassociation within the association Salicetum albae Issler 1924 leucojetosum aestivi subass. nov.

In Romania, the plant communities of the association Salicetum albae Issler 1924 are grouped in two subassociations - typicum and amorphaetosum fruticosae Morariu et Danciu 1970 [CHIFU \& IRIMIA, 2014; COLDEA, 2015] (Table 1).

The manner in which Salix alba phytocoenoses, with high abundance of the species Rubus caesius L. or Cornus sanguinea L s.1.., are grouped is questionable. In Slovenia, these phytocoenoses are separated in subassociations: cornetosum sanguinei Wendeberger Zelinka 1952 and rubetosum caesii (Soó 1958) Sílc 2003 of the association Salicetum albae Issler 1924 [SÍLC, 2003]. While in Romania, some researchers treat them as associations: Corno sanguinei-Salicetum albae Dihoru et al. 1966 em. Chifu et Irimia 2014 and Rubo caesiiSalicetum albae Doniță et Dihoru 1961 em. Doniță et al. 1966 [CHIFU \& IRIMIA, 2014]. On the other hand, other researchers do not recognize these coenotaxa [SEIBERT \& CONARD, 1992; IVAN \& al. 1993; NEUHÄSLOVÂ \& DOUDA, 2013; COLDEA, 2015]. Comparing the lists of the species of these coenotaxa and of the relevés from the Republic of Moldova, we conclude that it is appropriate to group these Salix alba forests into a single association - Salicetum albae Issler 1924 (Table 1) with the subassociations typicum, amorphaetosum fruticosae Morariu et Danciu 1970 and leucojetosum aestivi Pînzaru subass. nov. (Table 2). In the Salix alba riverside forests of the Republic of Moldova, Rubus caesius L. and Cornus sanguinea L. s.l. often grow abundantly together, for which reason, it has been proposed to consider them as characteristic species of the given association

We include the Salix alba L. phytocoenoses from the "Nemțeni" forest reserve, which was included some time ago in the association Salicetum albae-fragilis Issler 1926 [nome ambiguum, GAFTA \& al. 2008] by V. COVALI (2008), in the subassociation leucojetosum aestivi, because Leucojum aestivum, a species unregistered by the author, occurs in them (Table 2, rel. 17-18, h.1.). The description of the new coenotaxon is given below. 
As. Salicetum albae Issler 1924 leucojetosum aestivi Pînzaru subass. nov. hoc loco

T y p e h.l.: Table 2, rel. 9. (Figure 2)

Table synthetic h. 1.: Table 2, 19 relevés.

The total area of the subassociation is about 818 ha.

Syn.: Salicetum albae-fragilis auct. non Issler 1926, nome ambiguum: Covali, 2008

Locations: Altitude 20-25 m. Relief: the floodplain of Prut River. Soil: alluvial sandy, deep, rich in humus, with high trophicity. Climate - temperate continental, the average annual temperature is $10.5^{\circ} \mathrm{C}$, the average annual precipitation varies between $500 \mathrm{~mm}$ and $550 \mathrm{~mm}$ (Figure 6).

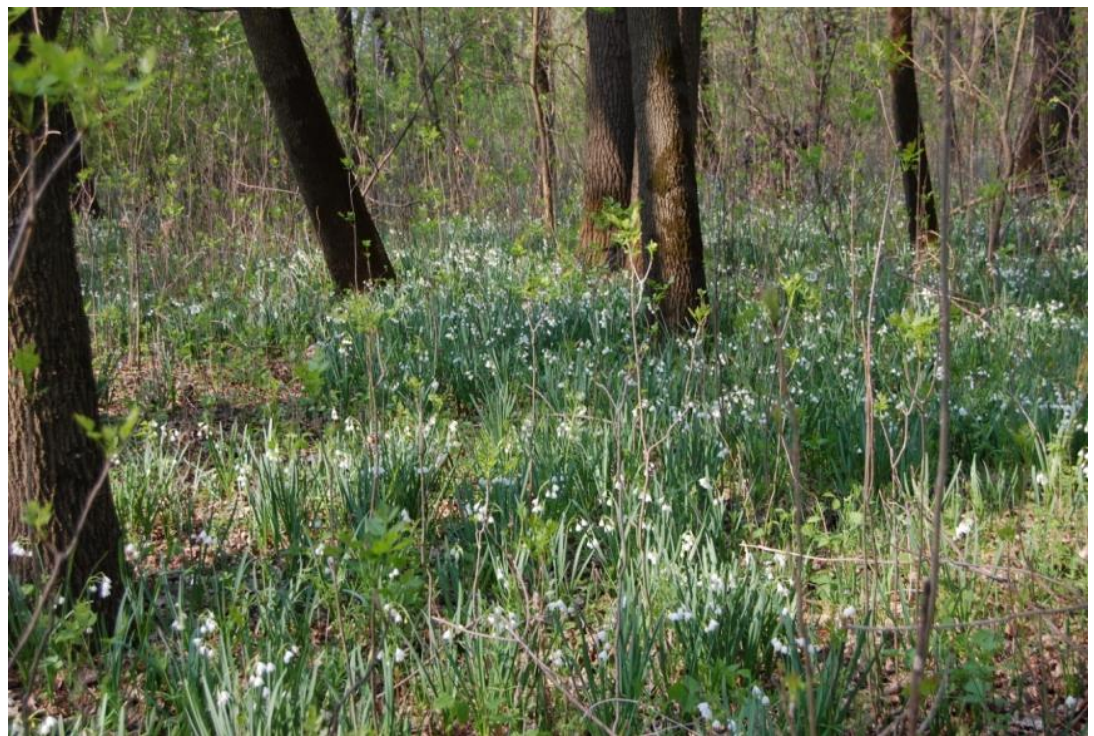

Figure 2. Salicetum albae leucojetosum aestivi subass. nov. (typus), Cioara commune, Hîncești district, 22.IV.2018

Characteristic species: Leucojum aestivum, Ficaria verna, Iris pseudacorus, Carex riparia, Salix alba.

Constant species: Populus alba, Fraxinus excelsior, Acer negundo, Cornus sanguinea, Crataegus monogyna, Rubus caesius, Glechoma hederacea, Lysimachia nummularia, Symphytum officinale, Stellaria media, Urtica dioica, Arctium tomentosum, Elymus repens, Galium aparine.

Structure: Vertically, three layers are distinguished in phytocoenoses (Figure 2, 3, 5):

1. The tree layer (A1), with a height of about (3-8)14-25 (-30) $\mathrm{m}$, the coverage of the canopy is about 0.6-0.8. This layer consists of the dominant species Salix alba, with the cover-abundance (AD) of (1)2-4(5) points, the diameter of the stems varies between (4-14) 20-80 (-100) cm. Accompanying species: Populus alba, Fraxinus excelsior, Salix fragilis, in some places Populus nigra, Quercus robur, Fraxinus americana. The layer A2 is poorly defined, with a height of about 5-10 m: Acer negundo, Pyrus pyraster, Malus sylvestris, Morus aba, Morus nigra, Ulmus minor, Ulmus glabra, Vitis sylvestris. 


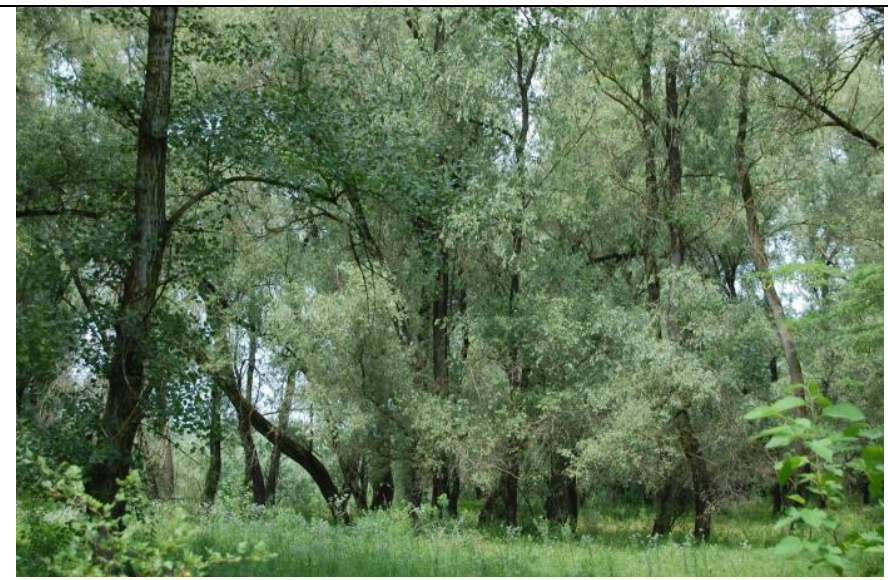

Figure 3. Salicetum albae-leucojetosum aestivi subass. nov. summer, 18.VI.2018

2. The shrub layer (B) is $1.5-3 \mathrm{~m}$ high, unevenly developed, with coverage 10-60 (80) \%. Constant species: Cornus sanguinea, Crataegus monogyna, in some places - Prunus spinosa, Rosa canina, Acer tataricum, Amorpha fruticosa; rarely Viburnum opulus, Coryllus avellana, Ligustrum vulgare, Euonymus europaeus. At the level of the shrub layer, there are abundant Acer negundo, Fraxinus excelsior, Fraxinus americana.

3. In the herbaceous cover layer (C), two synusiae can be observed: ephemeroidal and ephemeral. The spring vegetation is very uneven; Leucojum aestivum occurs in clusters, accompanied by Ficaria verna, Lamium purpureum, Anthriscus longirostris, Galium aparine, Stellaria media and Chaerophyllum temulum. The summer synusia is richer, with the general coverage varying from (0) 30 to $100 \%$, such species as Rubus caesius, Carex riparia, Glechoma hederacea, Elymus repens, Lysimachia nummularia and Poa palustris are dominant and constant, but Iris pseudacorus, Symphytum officinale, Arctium tomentosum and Valeriana officinalis occur sporadically but constantly.

Floristic composition: in 19 relevés, 113 species of vascular plants have been detected: 11 species are characteristic of the above-mentioned association, another 11 - to the alliance Salicion albae, 4 - Salicion triandrae, 7 - Salicetalia and Salicetea purpurae, 7 - Phragmitetea s.1., 17 - Molinio-Arrhenatheretea s.1., 16 - Querco-Fagetea s.1., 8 Rhamno-Prunetea s.1., 32 - Aliae.

Rare species protected by the state: Leucojum aestivum L. critically endangered (CR), included in the Red Book of R. Moldova [GHENDOV \& CIOCÂRLAN, 2015], Vitis sylvestris C.C.Gmel. endangered (EN), included in the Red Book of R. Moldova. [CANTEMIR \& ALEXANDROV, 2015] (Figure 4), Cephalanthera damasonium (Mill.) Druce, vulnerable (VU), included in the Red Book of R. Moldova [POSTOLACHE Gh \& JARDAN, 2015], Epipactis helleborine (L.) Crantz, near threatened (NT) Asparagus pseudoscaber Grecescu, near threatened (NT), Viburnum opulus L. near threatened (NT) [Legea privind fondul ariilor..., Anexa 3, D(a), 1998].

Phytocoenological diversity. Within the given subassociation, in some areas, there is a great abundance of the species Populus alba $\mathrm{L}$. $(\mathrm{AD}=3-4)$, which is proposed to be grouped in facies populosum albae (Table 2. rel. 3, 6, 7, 14, 17, 19, Figure 5). 
Range. The phytocoenoses of the subassociation leucojetosum aestivi occupy large areas in Leova (in the vicinity of Sărata-Răzeși commune) and Hîncești districts (in the vicinity of Cioara, Dancu, Călmăuți, Leușeni, Cotul Morii and Nemțeni communes).

Limiting factors. Clearcutting and uncontrolled logging lead to changes in the composition of the stands, sometimes willow forests are replaced by pure plantations of Gleditsia tricanthos L., Populus nigra L., Fraxinus excelsior L., and the sectors where Salix alba is partially eliminated are invaded by Acer negundo L. and Fraxinus americana L.

Conservation value. These plant communities are of great importance, they represent the only habitat in the Republic of Moldova where Leucojum aestivum L. grows.

Conservation status. The phytocoenoses of this subassociation are protected in the Dancu Nature Reserve (131.57 ha) and in the Nemțeni Nature Reserve (20.9 ha) [Legea privind fondul ariilor..., Anexa 4, A, 1998].

Protection measures. It has been proposed include the floodplain forests with the above-mentioned subassociation, found near Sărata-Răzeși, Leușeni and Cotul Morii, in the network of protected areas. During the ecological reconstruction activities, the structure and composition of the tree species characteristic of the association should be improved.

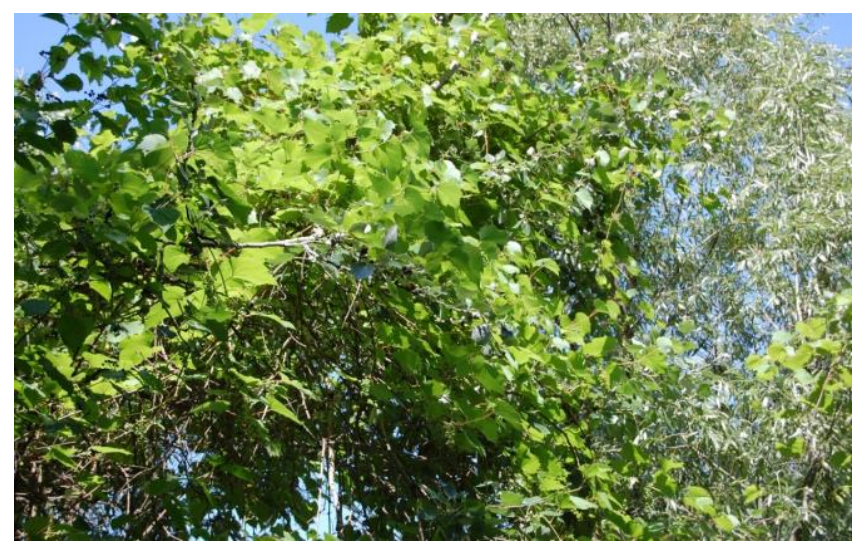

Figure 4. Vitis sylvestris C. C. Gmel in the forest near Cotul Morii commune, Hîncești district, 19.VI.2018

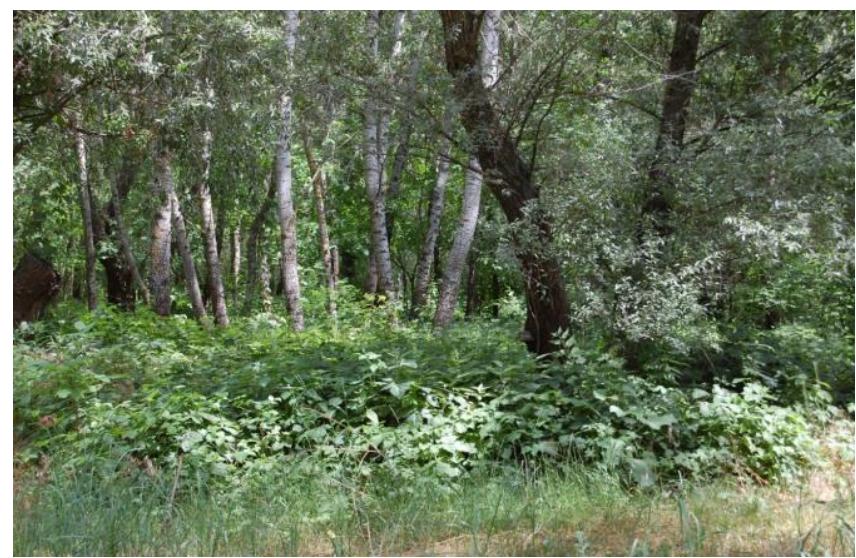

Figure 5. Facies populosum albae in the "Nemțeni" nature reserve, 10.VI.2018 


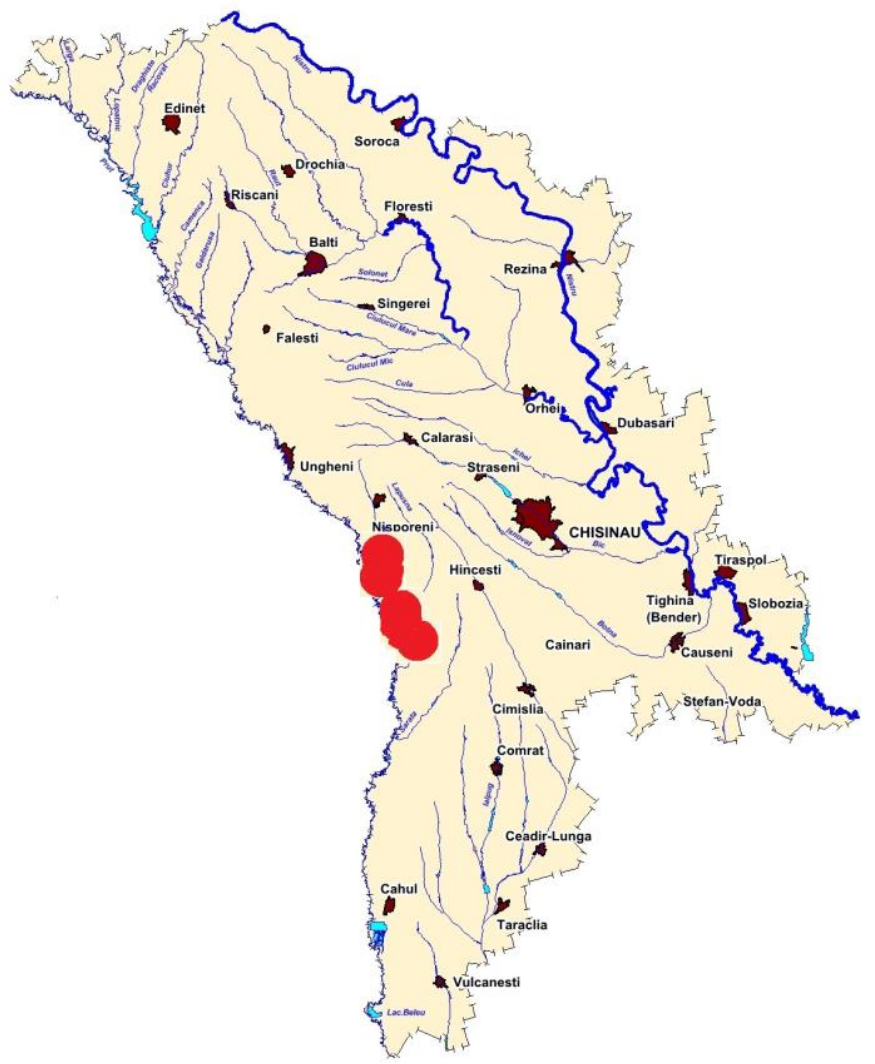

Figure 6. Locations of the subassociation leucojetosum aestivi subass. nov. in the Republic of Moldova

\section{Conclusions}

The subassociation leucojetosum aestivi Pînzaru represents rare mesohygrophylic phytocoenoses, and its entire range needs to be protected by the state. It is proposed to be included in the List of Rare Plant Associations of the Republic of Moldova.

The phytocoenoses with a great abundance of the species Populus alba L. and a rarer presence of the species Salix alba L. are proposed to be grouped in facies populosum albae (Table 2, rel. 3, 6, 7, 14, 17, 19).

The subassociation leucojetosum aestivi subass. nov. should be considered as part of the association Salicetum albae Issler 1924, the alliance Salicion albae Soó 1951, the order Salicetalia purpureae Moor 1958, cl. SALICETEA PURPUREAE Moor 1958.

\section{Notes on contributor}

Pavel PÎNZARU is a professor, $\mathrm{PhD}$, botanist with a special interest in phytocoenology, floristics and systematics. He has published over 150 scientific papers on the flora and vegetation of Bessarabia and Italy. He is a co-author of the Red Book of the Republic of Moldova $(2001,2015)$ and The Flora of Bessarabia (volume 1 and 2). 
SALICETUM ALBAE ISSLER 1924 LEUCOJETOSUM AESTIVI PÎNZARU SUBASS. NOV. IN...

\begin{tabular}{|c|c|c|c|c|c|c|c|c|c|c|c|}
\hline \multirow{6}{*}{$\begin{array}{l}\text { Subassociation: } \\
\text { Number of the column } \\
\text { Number of the relevés } \\
\text { Number of species } \\
\text { Constancy (K) or \% } \\
\text { Characteristic species }\end{array}$} & \multicolumn{9}{|c|}{$\mathbf{a}$} & \multicolumn{2}{|l|}{ b } \\
\hline & 1 & 2 & 3 & 4 & 5 & 6 & 7 & 8 & 9 & 10 & 11 \\
\hline & 6 & 12 & 16 & 26 & 86 & 116 & 108 & 83 & 28 & 19 & 18 \\
\hline & & & & & & & & & & & \\
\hline & $\mathrm{K}$ & $\mathrm{K}$ & $\mathrm{K}$ & $\bar{K}$ & $\%$ & $\mathrm{~K}$ & $\bar{K}$ & $\mathrm{~K}$ & $\mathrm{~K}$ & $\mathrm{~K}$ & $\%$ \\
\hline & & & & & & & & & & & \\
\hline Salix alba & $\mathrm{V}$ & $\mathrm{V}$ & $\mathrm{V}$ & $\mathrm{V}$ & 100 & $\mathrm{~V}$ & $\mathrm{~V}$ & $\mathrm{~V}$ & $\mathrm{~V}$ & II & 89 \\
\hline Salix fragilis & III & IV & IV & IV & 47 & III & V & I & $\mathrm{V}$ & I & 33 \\
\hline Cornus sanguinea & $\mathrm{V}$ & IV & IV & V & 27 & III & I & I & - & - & 28 \\
\hline Rubus caesius & IV & IV & IV & V & 5 & $\mathrm{~V}$ & III & V & V & IV & - \\
\hline Glechoma hederacea & - & I & II & V & 38 & II & II & I & IV & I & 22 \\
\hline Urtica dioica & V & IV & I & V & 50 & - & II & I & IV & - & 28 \\
\hline $\begin{array}{l}\text { Vitis sylvestris } \\
\text { Diff. subass. }\end{array}$ & - & I & I & IV & 6 & II & - & I & II & II & 44 \\
\hline Amorpha fruticosa & - & - & - & - & 9 & - & - & $\mathrm{I}$ & I & $\mathrm{V}$ & 72 \\
\hline Xanthium italicum & - & - & - & - & 1 & - & - & - & - & - & 17 \\
\hline Asparagus officinalis & - & - & - & - & 1 & - & - & - & - & - & 22 \\
\hline Asparagus tenuifolius & - & - & - & I & 1 & - & - & I & - & - & 17 \\
\hline Asparagus verticillatus & - & - & - & III & - & - & - & I & II & - & 17 \\
\hline Lycopus exaltatus & II & - & - & I & 2 & - & - & - & - & $\mathrm{I}$ & 28 \\
\hline Leucojum aestivum & - & - & - & - & - & - & - & - & - & - & - \\
\hline Iris pseudacorus & - & - & I & - & 10 & - & I & II & I & I & 17 \\
\hline Carex riparia & - & - & - & - & - & - & - & - & - & - & - \\
\hline Ficaria verna & - & - & - & I & - & - & - & - & I & - & - \\
\hline $\begin{array}{l}\text { Populus alba } \\
\text { Salicion albae }\end{array}$ & - & II & I & III & 51 & III & II & $\mathrm{I}$ & III & - & 33 \\
\hline Acer negundo & I & III & - & - & 1 & - & - & - & - & - & - \\
\hline Valeriana officinalis & - & - & - & - & - & - & I & - & I & - & - \\
\hline Viburnum opulus & II & I & II & III & 10 & II & I & I & III & - & 17 \\
\hline Humulus lupulus & III & IV & III & $\mathrm{V}$ & 35 & III & II & I & $\mathrm{V}$ & - & 28 \\
\hline Phalaris arundinacea & - & - & II & - & 8 & - & - & II & II & II & 11 \\
\hline Frangula alnus & - & II & II & IV & 8 & I & - & I & I & - & 28 \\
\hline Galium rubioides & - & - & - & - & - & - & - & $\mathrm{I}$ & I & I & - \\
\hline Silene bacifera & II & II & I & III & 8 & I & I & I & I & - & 11 \\
\hline Salix viminalis & - & I & I & - & 14 & I & II & I & I & - & - \\
\hline $\begin{array}{l}\text { Persicaria hydropiper } \\
\text { Salicion triandrae }\end{array}$ & - & II & III & - & 15 & - & I & II & - & - & - \\
\hline Galium aparine & III & II & II & II & 28 & I & - & II & - & I & - \\
\hline Lysimachia vulgaris & - & I & I & - & 13 & II & II & II & - & II & 28 \\
\hline Calystegia sepium & III & II & I & III & 37 & II & II & II & II & - & 28 \\
\hline Rumex obtusifolium & - & - & - & IV & 9 & - & I & - & III & - & 22 \\
\hline Salix triandra & - & I & I & II & 15 & I & I & I & - & - & 6 \\
\hline Salix pentandra & - & I & & - & - & - & I & - & II & - & - \\
\hline Aegopodium podagraria & II & - & - & III & 3 & II & I & - & I & - & 17 \\
\hline Epilobium hirsutum & - & - & - & - & - & - & I & - & - & - & - \\
\hline Elymus caninus & - & - & - & I & 5 & - & - & - & I & - & - \\
\hline Rumex crispus & - & - & - & - & I & - & - & I & - & - & - \\
\hline Salicion elaeagno-daph & & & & & & & & & & & \\
\hline Alnus incana & - & - & - & - & 12 & - & I & I & - & - & - \\
\hline Angelica sylvestris & II & - & - & I & 8 & I & - & I & - & - & - \\
\hline
\end{tabular}




\begin{tabular}{|c|c|c|c|c|c|c|c|c|c|c|c|}
\hline $\begin{array}{l}\text { Calamagrostis } \\
\text { pseudophragmites }\end{array}$ & - & - & - & - & 19 & - & - & - & - & - & - \\
\hline Chaerophyllum hirsutum & - & - & - & - & 5 & - & - & - & - & - & - \\
\hline Hippophaë rhamnoides & - & - & - & - & - & - & - & - & I & - & - \\
\hline Myricaria germanica & - & - & - & - & 1 & - & - & - & - & - & - \\
\hline Salix daphnoides & - & - & - & I & - & - & - & - & I & - & - \\
\hline Salix elaegnos & - & - & - & I & 6 & I & - & - & - & - & - \\
\hline Saponaria officinalis & - & - & - & - & 14 & I & I & - & - & - & - \\
\hline \multicolumn{12}{|c|}{ Salicetalia et Salicetea purpureae } \\
\hline Lysimachia nummularia & IV & III & III & IV & 33 & II & II & II & III & I & 39 \\
\hline Symphytum officinalis & III & II & II & IV & 34 & II & II & I & IV & II & 56 \\
\hline Poa palustris & - & II & & - & 5 & - & I & I & I & - & \\
\hline Populus nigra & - & II & I & $\mathrm{V}$ & 45 & III & I & I & III & I & 78 \\
\hline Solanum dulcamara & II & III & II & IV & 30 & I & I & - & IV & I & 33 \\
\hline Salix purpurea & - & I & I & II & 16 & - & II & 1 & I & - & 11 \\
\hline Rumex sanguineus & I & I & $\mathrm{I}$ & III & 3 & I & I & $\mathrm{I}$ & III & - & 22 \\
\hline Anthriscus sylvestris & - & II & II & I & - & - & I & - & - & - & - \\
\hline Tamarix ramosissima & - & & $\mathrm{I}$ & - & 5 & - & - & - & - & - & - \\
\hline Ranunculus repens & - & I & III & - & 47 & - & - & - & - & - & 50 \\
\hline Stellaria aquatica & - & - & - & $\mathrm{V}$ & 23 & - & I & II & $\mathrm{V}$ & I & 33 \\
\hline $\begin{array}{l}\text { Periploca graeca } \\
\quad \text { Phragmitetea s.l. }\end{array}$ & - & - & - & - & - & - & - & II & - & - & - \\
\hline Phragmites australis & II & I & I & I & 13 & - & I & I & I & III & 44 \\
\hline Carex melanostachya & I & - & - & - & - & - & - & - & - & - & - \\
\hline Inula helenium & II & - & - & - & - & - & I & - & - & - & - \\
\hline Eupatorium cannabinum & I & I & - & II & 12 & I & I & I & I & I & 22 \\
\hline Alisma plantago-aquatica & - & - & - & - & - & - & 1 & - & - & - & - \\
\hline Alopecurus aequalis & - & - & - & - & & - & - & $\mathrm{I}$ & - & - & - \\
\hline Bidens cernua & - & - & $\mathrm{I}$ & - & - & - & - & I & - & - & - \\
\hline Bidens tripartita & - & I & III & I & 22 & - & $\mathrm{I}$ & IV & I & I & 22 \\
\hline Bolboschoenus maritimus & - & - & - & - & - & - & - & I & - & I & - \\
\hline Caltha palustris & - & - & - & - & - & - & - & I & - & - & - \\
\hline Carex acutiformis & - & - & - & - & - & - & I & I & I & IV & - \\
\hline Carex vulpina & - & I & $\mathrm{I}$ & - & - & - & - & I & I & - & - \\
\hline Cyperus glomeratus & - & - & - & - & - & - & - & I & - & - & - \\
\hline Echinochloa crus-galli & - & I & II & I & - & - & - & $\mathrm{I}$ & I & - & 11 \\
\hline Eleocharis palustris & - & - & - & - & - & - & I & $\mathrm{I}$ & - & - & - \\
\hline Epilobium parviflorum & - & - & - & - & - & - & I & - & - & - & - \\
\hline Filipendula ulmaria & - & - & - & - & - & - & I & - & - & - & - \\
\hline Galium palustre & - & - & - & - & 3 & - & I & IV & I & II & - \\
\hline Glyceria fluitans & - & - & - & - & - & - & - & I & - & - & - \\
\hline Glyceria maxima & - & - & $\mathrm{I}$ & - & - & - & I & $\mathrm{I}$ & - & - & - \\
\hline Glycyrrhiza echinata & - & - & - & - & 6 & I & - & $\mathrm{I}$ & - & - & 17 \\
\hline Leersia oryzoides & - & - & - & - & - & - & - & $\mathrm{I}$ & I & - & - \\
\hline Lycopus europaeus & - & I & $\mathrm{I}$ & I & - & - & I & III & - & II & - \\
\hline Lythrum salicaria & I & I & I & - & 35 & - & I & III & - & II & 17 \\
\hline Mentha aquatica & - & - & I & - & 12 & - & I & - & I & I & 6 \\
\hline Myosotis scorpioides & - & I & - & - & - & I & II & I & I & I & - \\
\hline Oenanthe aquatica & - & - & - & - & 5 & - & - & I & - & I & 11 \\
\hline Oenanthe silaifolia & - & - & - & - & - & - & - & - & I & - & - \\
\hline Persicaria lapatifolia & - & - & - & I & - & - & - & I & - & - & 28 \\
\hline Persicaria maculosa & - & - & - & - & 9 & - & I & - & - & - & - \\
\hline
\end{tabular}




\begin{tabular}{|c|c|c|c|c|c|c|c|c|c|c|c|}
\hline Persicaria mitis & - & - & - & - & - & - & - & I & - & - & - \\
\hline Ranunculus scleratus & - & - & - & - & - & - & - & I & - & - & - \\
\hline Rorippa amphybia & - & - & - & I & 13 & - & - & I & - & - & - \\
\hline Rumex conglomeratus & - & I & I & I & - & - & I & I & I & - & - \\
\hline Rumex hydrolapathum & - & - & - & - & - & - & - & - & - & I & - \\
\hline Sagittaria sagittifolia & - & - & - & - & - & - & - & I & - & - & - \\
\hline Scirpus sylvaticus & II & - & - & - & - & - & - & I & I & - & - \\
\hline Scutellaria galericulata & - & I & I & I & 21 & - & - & $\mathrm{I}$ & I & - & - \\
\hline Senecio paludosus & - & - & & - & - & - & - & I & - & - & - \\
\hline Sium sisarum & I & - & - & - & - & - & I & - & - & - & - \\
\hline Sonchus oleraceus & - & - & - & - & - & - & - & I & - & - & - \\
\hline Sparganium erectum & - & - & - & - & - & - & - & I & - & - & - \\
\hline Stachys palustris & - & - & - & - & 12 & - & I & IV & I & - & 11 \\
\hline Teucrium scordium & - & - & - & - & - & - & - & - & - & II & - \\
\hline Typha angustifolia & - & - & - & - & - & - & - & I & - & I & - \\
\hline $\begin{array}{l}\text { Veronica anagalis- } \\
\text { aquatica }\end{array}$ & - & - & - & I & - & - & - & - & I & - & - \\
\hline \multicolumn{12}{|c|}{ Molinio-Arrhenatheretea s.l. } \\
\hline Taraxacum camylodes & - & - & I & - & 12 & - & I & I & I & I & 6 \\
\hline Euphorbia lucida & - & - & - & I & - & - & - & - & I & I & - \\
\hline Potentilla reptans & - & I & I & - & - & - & II & I & I & I & - \\
\hline Carex hirta & - & - & - & - & 9 & - & I & I & I & I & - \\
\hline Daucus carota & - & - & I & - & 13 & - & I & I & I & - & - \\
\hline Prunella vulgaris & - & - & I & II & 7 & - & I & - & - & - & 17 \\
\hline Rorippa sylvestris & - & - & I & II & 12 & - & I & I & I & II & 39 \\
\hline Scutellaria hastifolia & I & - & I & I & - & - & - & I & - & - & - \\
\hline Thalictrum lucidum & - & - & - & - & - & - & - & $\mathrm{I}$ & - & - & - \\
\hline Trifolium repens & - & - & I & - & 15 & 15 & II & - & I & - & - \\
\hline Cerastium holosteoides & - & - & - & - & - & - & II & - & - & - & 17 \\
\hline Equisetum arvense & II & II & - & - & 20 & - & II & I & I & II & 11 \\
\hline $\begin{array}{l}\text { Ranunculus } \\
\text { polyanthemos }\end{array}$ & - & - & - & - & - & - & I & - & - & - & - \\
\hline Trifolium campestre & - & - & - & - & - & - & I & - & I & - & - \\
\hline Trifolium pratense & - & I & - & - & 1 & - & - & - & - & - & - \\
\hline Achillea millefolium & - & - & - & - & 1 & - & I & I & - & - & - \\
\hline Agrostis stolonifera & - & II & I & III & 30 & - & I & III & III & II & 72 \\
\hline Alopecurus geniculatus & - & - & - & - & - & - & - & - & I & - & - \\
\hline Alopecurus pratensis & - & - & - & - & - & - & I & - & - & - & - \\
\hline Althaea officinalis & - & II & II & IV & 10 & I & I & I & III & - & 28 \\
\hline Anthoxanthum odoratum & - & - & - & - & - & - & I & - & - & - & - \\
\hline Barbarea vulgaris & - & - & - & - & - & - & I & - & - & - & - \\
\hline Bellis perennis & - & - & - & - & - & - & I & - & I & - & - \\
\hline Briza media & - & - & - & - & - & - & I & - & - & - & - \\
\hline Carex brizoides & - & - & - & - & - & - & I & - & - & - & - \\
\hline Cardamine pratensis & - & - & - & - & - & - & - & I & - & - & - \\
\hline Centaurea phrygia & - & - & & - & - & - & I & - & - & - & - \\
\hline Centaurium erythraea & - & - & - & - & - & - & - & - & I & - & - \\
\hline Centaurium pulchellum & & & & I & & & & & & & \\
\hline Dactylis glomerata & - & I & I & - & - & - & I & - & - & - & - \\
\hline Equisetum palustre & - & - & - & I & - & I & - & II & - & - & 28 \\
\hline Equisetum telmateia & III & - & - & - & - & - & I & - & - & - & - \\
\hline Euphorbia palustris & - & - & I & I & - & I & - & - & - & I & - \\
\hline
\end{tabular}




\begin{tabular}{|c|c|c|c|c|c|c|c|c|c|c|c|}
\hline & & & & & & & & & & & \\
\hline Festuca pratensis & - & - & - & - & - & - & I & - & - & - & - \\
\hline Galega officinalis & - & - & - & - & - & - & I & I & - & - & - \\
\hline Geranium pratense & - & - & - & - & 3 & - & I & - & - & - & - \\
\hline Heracleum sphondylium & - & - & - & - & 7 & - & II & - & - & - & - \\
\hline Holcus lanatus & - & - & - & - & 1 & - & I & - & I & - & - \\
\hline Inula britanica & - & - & - & - & 10 & - & II & I & - & - & - \\
\hline Juncus articulatus & - & - & - & - & - & - & I & - & - & - & - \\
\hline Juncus effusus & - & - & - & - & - & - & I & - & - & - & - \\
\hline Mentha longifolia & - & I & I & I & 16 & - & I & I & I & - & 11 \\
\hline Plantago major & - & I & - & I & - & - & - & - & - & - & - \\
\hline Peucedanum carvifolium & - & I & III & - & - & - & - & - & I & - & - \\
\hline Leucanthemum vulgare & - & - & - & - & 3 & - & I & I & - & - & - \\
\hline Lolium perenne & - & - & - & - & 1 & - & I & - & - & - & - \\
\hline Luzula campestris & - & - & - & - & - & - & I & - & - & - & - \\
\hline Lysimachia punctata & - & - & - & - & - & - & - & - & I & - & - \\
\hline Lythrum virgatum & - & - & - & - & - & - & I & - & - & - & - \\
\hline Medicago lupulina & - & - & - & - & 2 & - & - & - & - & - & - \\
\hline Mentha arvensis & - & - & - & - & - & - & I & - & I & - & - \\
\hline $\begin{array}{l}\text { Pastinaca sativa var. } \\
\text { sylvestris }\end{array}$ & - & - & - & - & - & - & I & I & - & - & - \\
\hline Petasites hybridus & IV & - & - & - & - & - & I & I & - & - & - \\
\hline Picris hieracioides & - & - & - & - & - & - & I & - & - & - & - \\
\hline Poa pratensis & - & - & - & - & - & - & I & - & I & - & - \\
\hline Poa sylvicola & - & - & - & - & - & - & - & - & I & I & - \\
\hline Poa trivialis & - & - & - & - & 5 & - & I & - & $\mathrm{I}$ & - & - \\
\hline Potentilla anserina & - & - & - & I & - & - & I & - & - & - & - \\
\hline Ranunculus acris & - & - & - & - & 12 & - & I & - & - & - & - \\
\hline Ranunculus repens & - & - & - & III & - & - & III & II & II & II & - \\
\hline Rhinanthus minor & - & - & - & - & - & - & I & - & - & - & - \\
\hline Rumex acetosa & - & - & - & - & - & - & I & - & - & - & - \\
\hline Rumex dentatus & - & - & - & - & 2 & - & - & - & - & - & - \\
\hline Silene flos-cuculi & - & - & - & - & - & - & I & - & - & - & - \\
\hline Silene vulgaris & - & - & - & I & - & - & - & I & - & - & - \\
\hline Stellaria graminea & - & - & - & - & - & - & I & - & - & - & - \\
\hline Thalictrum flavum & - & - & - & - & - & - & - & I & - & - & - \\
\hline Thalictrum simplex & - & - & - & - & - & - & I & - & - & - & - \\
\hline Trifolium hybridum & - & - & - & - & - & - & I & - & - & - & - \\
\hline Trisetum flavescens & - & - & - & - & - & - & I & - & - & - & - \\
\hline $\begin{array}{l}\text { Vicia cracca } \\
\text { Querco-Fagetea s.l. }\end{array}$ & - & - & - & - & 13 & - & I & I & - & I & - \\
\hline Fraxinus excelsior & III & - & - & - & - & - & I & - & - & - & - \\
\hline Quercus robur & - & - & - & I & - & - & - & - & - & - & - \\
\hline Chaerophyllum temulum & - & - & - & - & - & - & - & - & I & - & - \\
\hline Malus sylvestris & I & - & - & - & - & - & - & - & - & - & - \\
\hline Epipactis helleborine & $\mathrm{I}$ & - & - & - & - & - & - & - & - & - & - \\
\hline Convallaria majalis & I & - & - & - & - & - & - & - & - & - & - \\
\hline Ulmus minor & - & - & - & III & 12 & I & - & - & I & I & 11 \\
\hline Ulmus glabra & - & - & - & - & - & - & - & I & - & - & - \\
\hline Euonymus europaeus & $\mathrm{I}$ & I & - & II & 10 & I & - & - & I & - & 22 \\
\hline Geum urbanum & II & I & I & I & 8 & - & I & - & I & - & - \\
\hline Lapsana communis & - & - & - & - & - & - & I & I & I & - & - \\
\hline Salix cinerea & III & I & - & I & - & - & II & - & - & - & - \\
\hline
\end{tabular}




\begin{tabular}{|c|c|c|c|c|c|c|c|c|c|c|c|}
\hline Populus tremula & III & - & - & - & - & - & I & - & - & - & - \\
\hline Cerasus avium & I & - & - & I & - & - & - & - & - & - & - \\
\hline Acer campestre & - & I & - & II & - & - & - & - & I & - & - \\
\hline Acer pseudoplatanus & - & - & - & - & - & - & I & - & - & - & - \\
\hline Ajuga reptans & - & - & I & - & - & - & - & - & - & - & - \\
\hline Alnus glutinosa & - & - & - & - & - & II & - & I & I & - & - \\
\hline Arctium nemorosum & - & - & - & - & - & - & - & - & I & - & - \\
\hline Athyrium filix-femina & - & - & - & - & - & - & I & - & - & - & - \\
\hline $\begin{array}{l}\text { Brachypodium } \\
\text { sylvaticum }\end{array}$ & II & - & I & I & 8 & - & I & - & - & - & - \\
\hline Bromus benekenii & $\mathrm{I}$ & - & - & - & - & - & - & - & - & - & - \\
\hline Campanula persicifolia & - & - & - & I & - & - & - & - & - & - & - \\
\hline Campanula rapunculoides & - & - & - & I & - & - & - & - & - & - & - \\
\hline Campanula trachelium & - & - & - & - & - & - & - & - & I & - & - \\
\hline Carex pendula & I & - & - & - & - & - & - & - & - & - & - \\
\hline Carex remota & - & II & - & - & - & - & - & - & I & - & - \\
\hline Cardamine impatiens & I & - & - & - & - & I & - & I & - & - & - \\
\hline $\begin{array}{l}\text { Chrysosplenium } \\
\text { alternifolium }\end{array}$ & - & - & - & - & - & - & I & - & - & - & - \\
\hline Dryopteris filix-mas & - & - & - & - & - & - & I & - & - & - & - \\
\hline Equisetum hyemale & - & - & - & I & - & - & - & - & I & - & - \\
\hline Festuca gigantea & - & - & - & I & - & - & - & - & I & - & - \\
\hline Fraxinus oxycarpa & - & - & - & I & 10 & - & - & - & I & - & - \\
\hline Fraxinus pallisae & - & - & I & - & - & - & - & - & - & - & - \\
\hline Geranium phaeum & II & - & I & - & - & - & I & - & - & - & - \\
\hline Glechoma hirsuta & I & - & - & - & - & - & - & - & - & - & - \\
\hline Impatiens noli-tangere & - & - & - & - & - & - & 1 & - & - & - & - \\
\hline Lactuca muralis & - & - & - & - & I & - & I & - & - & - & - \\
\hline Lamium galeobdolon & II & - & - & - & - & - & I & - & - & - & - \\
\hline Lonicera xylosteum & - & - & - & I & - & - & I & - & - & - & - \\
\hline Matteucia struthiopteris & - & - & - & - & - & - & I & - & - & - & - \\
\hline Myosotis sparsiflora & - & - & - & - & - & - & - & - & I & - & - \\
\hline Platanthera bifolia & - & - & - & - & - & - & - & - & I & - & - \\
\hline Poa nemoralis & - & - & - & - & - & - & I & - & - & - & - \\
\hline Pulmonaria officinalis & - & I & - & - & - & - & - & - & - & - & - \\
\hline Ribes uva-crispi & - & - & - & - & - & - & I & - & - & - & - \\
\hline Salvia glutinosa & I & - & - & - & 2 & - & - & - & - & - & - \\
\hline Sambucus nigra & III & II & I & II & 30 & I & - & - & - & - & 11 \\
\hline Scrophularia nodosa & - & - & - & - & 6 & - & I & I & I & - & - \\
\hline Stachys sylvatica & II & - & - & - & - & - & I & - & - & - & - \\
\hline Staphylea pinnata & - & - & - & - & - & - & - & I & - & - & - \\
\hline Stellaria nemorum & - & - & - & I & - & - & I & - & I & - & - \\
\hline Tanacetum corymbosum & - & - & - & I & - & - & - & - & - & - & - \\
\hline Telekia speciosa & - & - & - & - & - & - & I & - & - & - & - \\
\hline Tilia cordata & - & - & - & I & - & - & - & - & - & - & - \\
\hline Tilia tomentosa & - & - & - & - & - & - & - & I & - & - & - \\
\hline Ulmus laevis & $\mathrm{I}$ & - & - & - & - & - & - & I & - & - & - \\
\hline Ulmus procera & - & & - & - & - & - & - & - & I & - & - \\
\hline Viola odorata & - & - & - & I & - & - & - & - & - & - & - \\
\hline $\begin{array}{l}\text { Viscum album } \\
\text { Rhamno-Prunetea s.l. }\end{array}$ & - & - & - & - & - & - & - & - & $\mathrm{I}$ & - & - \\
\hline Crataegus monogyna & III & I & - & IV & 35 & I & - & I & III & - & 11 \\
\hline
\end{tabular}


PAVEL PÎNZARU

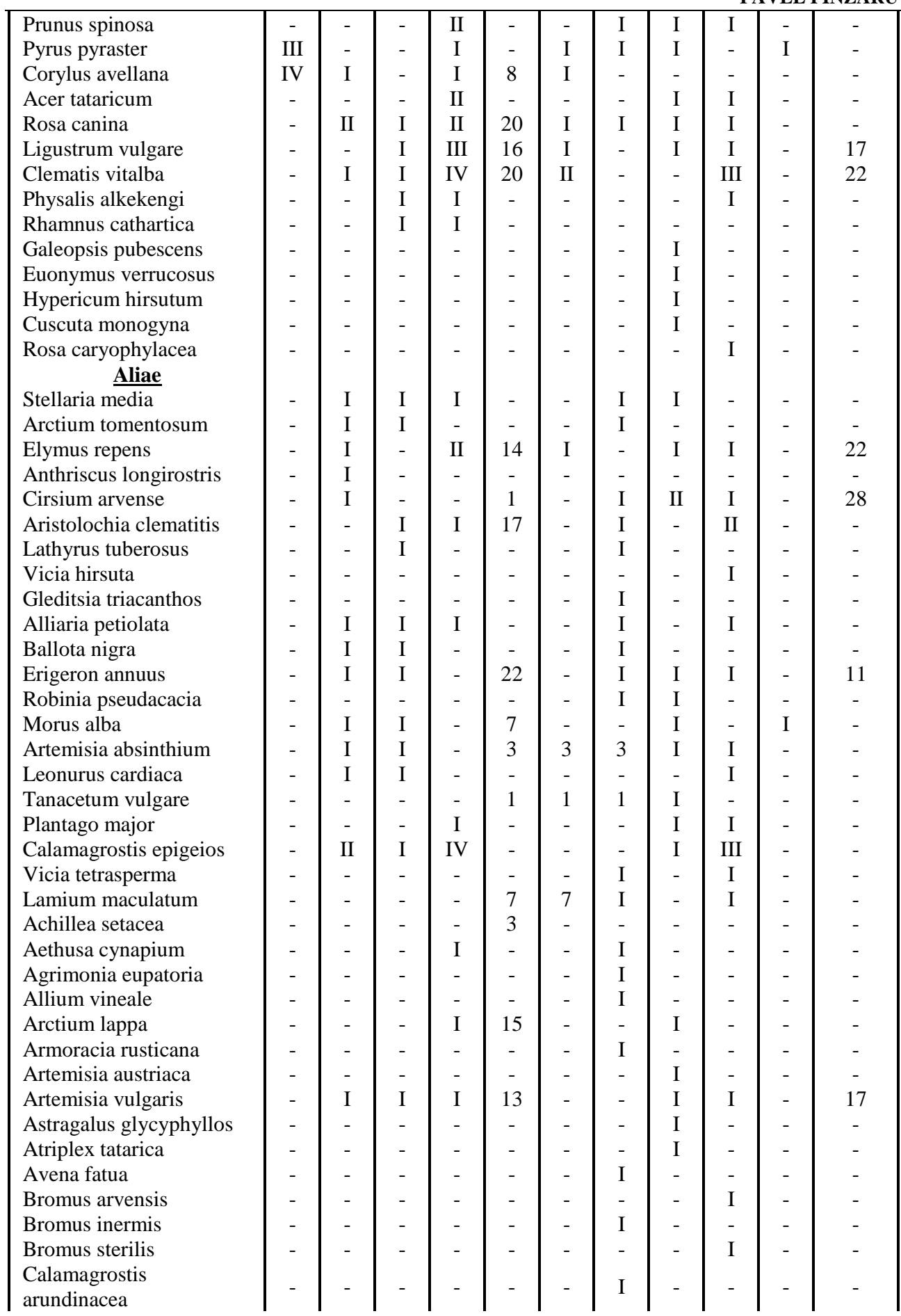




\begin{tabular}{|c|c|c|c|c|c|c|c|c|c|c|c|}
\hline Campanula abietina & - & - & - & - & - & - & I & - & - & - & - \\
\hline Cannabis sativa & - & I & I & - & - & - & - & - & - & - & - \\
\hline Cardamine amara & - & - & - & - & - & - & - & I & - & - & - \\
\hline Carduus acanthoides & - & - & - & - & 5 & - & I & - & - & - & - \\
\hline Carduus crispus & - & II & I & II & 7 & - & I & - & I & - & 22 \\
\hline Centaurea micranthos & - & - & - & - & - & - & I & - & - & - & - \\
\hline $\begin{array}{l}\text { Chaerophyllum } \\
\text { aromaticum }\end{array}$ & - & - & - & - & - & - & I & - & - & - & - \\
\hline Chaerophyllum bulbosum & - & - & I & - & - & - & - & - & - & - & - \\
\hline Chelidonium majus & - & - & - & I & - & - & I & - & - & - & - \\
\hline Chenopodium album & - & - & - & - & - & - & - & I & - & - & - \\
\hline Cichorium intybus & - & - & - & I & 1 & 1 & I & - & - & - & - \\
\hline Cirsium vulgare & - & I & I & - & 6 & - & I & I & I & - & - \\
\hline Clinopodium vulgare & - & - & - & I & - & - & I & - & - & - & - \\
\hline Conium maculatum & - & - & - & I & - & - & I & - & I & - & - \\
\hline Convolvulus arvensis & - & - & - & - & - & - & I & - & - & - & - \\
\hline Cruciata laevipes & - & - & - & - & - & I & - & I & - & - & - \\
\hline Cuscuta lupuliformis & - & - & - & I & - & - & - & - & I & - & - \\
\hline Cynodon dactylon & - & - & - & - & 1 & - & - & I & - & I & - \\
\hline Dipsacus fullonum & II & I & - & - & - & - & - & - & I & - & - \\
\hline Dipsacus laciniatus & - & - & - & - & - & - & I & - & I & - & - \\
\hline Dipsacus pilosus & - & I & - & - & - & - & - & - & - & - & - \\
\hline Dysphania botrys & - & - & - & - & - & - & - & I & - & - & - \\
\hline Echinocystis lobata & - & - & - & - & 16 & - & I & I & - & - & - \\
\hline $\begin{array}{l}\text { Echinops } \\
\text { sphaerocephalus }\end{array}$ & - & - & - & - & - & - & - & I & - & - & - \\
\hline Echium vulgare & - & - & - & - & - & - & I & - & - & - & - \\
\hline Erigeron acer & - & - & - & - & 9 & - & - & - & - & - & 11 \\
\hline Erigeron canadensis & - & - & - & - & 1 & - & I & I & I & - & - \\
\hline Euphorbia cyparissias & - & - & - & - & 13 & - & I & I & - & - & - \\
\hline Fallopia dumetorum & - & - & - & I & 5 & - & - & - & - & - & 11 \\
\hline Festuca valesiaca & - & - & - & - & - & - & I & - & - & - & - \\
\hline Filipendula vulgaris & - & - & - & - & - & - & - & - & I & - & - \\
\hline Fragaria vesca & - & - & - & I & - & - & I & - & - & - & - \\
\hline Fraxinus pennsylvanica & - & - & - & - & - & - & - & II & - & - & - \\
\hline Galeopsis speciosa & - & - & - & - & - & - & - & I & I & - & - \\
\hline Galeopsis tetrahit & II & - & - & - & - & - & I & - & - & - & - \\
\hline Galinsoga parviflora & - & - & - & - & - & - & I & - & - & - & - \\
\hline Galium verum & - & - & - & - & - & - & I & - & - & - & - \\
\hline Gentiana asclepiadea & - & - & - & - & - & - & I & - & - & - & - \\
\hline Geranium robertianum & - & - & - & - & - & - & I & - & - & - & - \\
\hline Helianthus tuberosus & - & - & - & - & - & - & I & - & - & - & - \\
\hline Hieracium aurantiacum & - & - & - & - & - & - & I & - & - & - & - \\
\hline Hypericum perforatum & - & - & - & - & - & - & - & I & I & - & - \\
\hline Lactuca saligna & - & - & - & - & - & - & - & I & - & - & - \\
\hline Lactuca seriola & - & - & I & - & - & - & - & I & - & - & - \\
\hline Lamium album & - & - & - & - & - & - & I & - & - & - & - \\
\hline Leonurus marrubiastrum & - & - & - & - & - & - & - & I & - & - & - \\
\hline Linaria vulgaris & - & - & - & - & - & - & I & - & - & - & - \\
\hline Lipandra polysperma & - & - & - & - & - & - & - & I & - & - & - \\
\hline Lithospermum officinale & - & - & - & - & - & - & - & - & I & - & - \\
\hline Lotus tenuis & - & - & - & - & - & - & I & - & - & - & - \\
\hline
\end{tabular}




\begin{tabular}{|c|c|c|c|c|c|c|c|c|c|c|c|}
\hline Medicago falcata & - & - & - & - & - & - & $\mathrm{I}$ & - & - & - & - \\
\hline Mentha pulegium & - & - & - & - & - & - & I & I & - & - & - \\
\hline Melilotus officinalis & - & - & - & - & - & - & - & I & - & - & - \\
\hline Oenothera biennis & - & I & - & II & 15 & I & - & - & II & - & 6 \\
\hline Oxalis stricta & - & - & - & - & - & - & - & I & - & - & - \\
\hline Oxybasis ubrica & - & - & - & - & - & - & - & I & - & - & - \\
\hline Parietaria officinalis & I & - & - & - & - & - & - & - & I & - & - \\
\hline Plantago lanceolata & - & - & - & - & 10 & - & I & - & - & - & - \\
\hline Plantago media & - & - & - & - & - & - & - & - & I & - & - \\
\hline Poa annua & - & - & - & I & 2 & - & - & - & - & - & - \\
\hline Polygonum aviculare & - & - & - & - & - & - & - & I & - & - & - \\
\hline Potentilla argentea & - & - & - & - & - & - & I & - & - & - & - \\
\hline Potentilla recta & - & - & - & - & - & - & - & - & I & - & - \\
\hline Potentilla supina & - & - & - & I & - & - & - & - & - & - & - \\
\hline Reseda lutea & - & - & - & - & - & - & I & - & - & - & - \\
\hline Rumex pulcher & - & - & - & - & - & - & - & I & - & - & - \\
\hline Sambucus ebulus & - & I & I & - & 15 & - & - & - & I & - & - \\
\hline Senecio germanicus & - & - & - & - & - & - & - & I & - & - & - \\
\hline Senecio sylvaticus & - & - & - & - & - & - & - & I & - & - & - \\
\hline Setaria pumila & - & - & - & - & - & - & I & - & - & - & - \\
\hline Setaria viridis & - & - & - & - & 1 & - & - & - & - & - & - \\
\hline Silene alba & - & - & - & - & 8 & - & - & - & - & - & 6 \\
\hline Solanum nigrum & - & - & - & - & 9 & - & I & - & - & - & - \\
\hline Sonchus arvensis & - & I & - & - & 6 & - & - & - & - & - & 6 \\
\hline Sonchus asper & - & & - & - & - & - & I & I & - & - & - \\
\hline Torilis japonica & - & - & - & I & - & - & - & I & I & - & - \\
\hline Trifolium fragiferum & - & - & - & - & - & - & I & - & - & - & - \\
\hline Tussilago farfara & - & I & I & - & 3 & - & I & - & I & - & - \\
\hline Verbena officinalis & - & - & - & - & - & - & I & I & - & - & - \\
\hline Veronica chamaedrys & - & - & - & - & - & - & I & - & - & - & - \\
\hline Vicia pannonica & - & - & - & - & - & - & - & - & I & - & - \\
\hline Xanthium spinosum & - & - & - & - & - & - & - & I & - & - & - \\
\hline Xanthium strumarium & - & I & I & - & 6 & - & I & $\mathrm{I}$ & II & - & 6 \\
\hline
\end{tabular}

Ass. Saliceum albae Issler 1924 [= Salicetum albae Issler 1926]:

a. typicum [= Rubo caesii-Salicetum albae Doniță et Dihoru 1961 em. Doniță et al. 1966; Corno sanguinei-Salicetum albae Dihoru et al. $1966 \mathrm{em}$. Chifu et Irimia 2014]:

Col. 1.6 rel. POSTOLACHE Gh \& CHIRTOACĂ, 2005 (Salicetum albae Issler 1924)

Col. 2. 12 rel. POSTOLACHE Gh. 2017 (Salicetum albae Issler 1926)

Col. 3. 16 rel. POSTOLACHE Gh. \& POSTOLACHE D., 2012 (Salicetum albae Issler 1926)

Col. 4. 26 rel.TOFAN-BURAC \& CHIFU, 2002 (Salicetum albae Isseler 1924)

Col. 5. 86 rel. COLDEA, 2015 (Salicetum albae Issler 1926 typicum)

Col. 6. 116 rel. IVAN \& al. 1993 (Salicetum albae Issler 1924)

Col. 7. 108 rel. CHIFU \& IRIMIA, 2014 (Salicetum albae Issler 1926 typicum)

Col. 8. 83 rel. CHIFU \& IRIMIA 2014 (Ass. Rubo caesii-Salicetum albae Doniță et Dihoru 1961 em. Doniță et al. 1966)

Col. 9. 28 rel. CHIFU \& IRIMIA2014 (Ass. Corno sanguinei-Salicetum albae Dihoru et al. 1966 em. Chifu et Irimia 2014) b. subass. amorphaetosum fruticosae Morariu et Danciu 1970

Col. 10. 19 rel. CHIFU \& IRIMIA, 2014

Col. 11. 18 rel. COLDEA, 2015 
SALICETUM ALBAE ISSLER 1924 LEUCOJETOSUM AESTIVI PÎNZARU SUBASS. NOV. IN...

Table 2. Ass. Salicetum albae Issler 1924 leucojetosum aestivi subass. nov.

\begin{tabular}{|c|c|c|c|c|c|c|c|c|c|c|c|c|c|c|c|c|c|c|c|c|}
\hline Relevé no. & 1 & 2 & 3 & 4 & 5 & 6 & 7 & $\overline{8}$ & $* 9$ & 10 & 11 & 12 & 13 & 14 & 15 & 16 & 17 & 18 & 19 & $\bar{K}$ \\
\hline Surface of relevé $\left(\mathrm{m}^{2}\right)$ & 600 & 600 & 600 & 600 & 600 & 600 & 600 & 600 & 600 & 600 & 600 & 600 & 600 & 600 & 600 & 600 & 600 & 600 & 600 & \\
\hline Altitudine & 20 & 20 & 20 & 20 & 20 & 20 & 20 & 20 & 20 & 20 & 20 & 20 & 20 & 20 & 20 & 22 & 22 & 24 & 25 & \\
\hline Consistency & 0,6 & 0,6 & 0,7 & 0,6 & 0,6 & 0,7 & 0,7 & 0,6 & 0,6 & 0,8 & 0,6 & 0,6 & 0,7 & 0,7 & 0,8 & 0,6 & 0,8 & 0,6 & 0,7 & \\
\hline & $12-$ & $16-$ & $18-$ & $15-$ & $19-$ & $18-$ & $17-$ & & $14-$ & & $21-$ & $6-$ & $19-$ & $19-$ & $17-$ & $24-$ & & $17-$ & $26-$ & \\
\hline Tree height (m) & 16 & 20 & 21 & 17 & 22 & 25 & 24 & 14 & 16 & $3-5$ & 24 & 24 & 24 & 25 & 19 & 27 & $8-9$ & 21 & 30 & \\
\hline & $24-$ & $24-$ & $22-$ & $20-$ & $28-$ & $26-$ & $24-$ & & $16-$ & & $32-$ & $10-$ & $26-$ & $30-$ & $24-$ & $52-$ & $10-$ & $30-$ & $30-$ & \\
\hline Tree diametr $(\mathrm{cm})$ & 36 & 34 & 30 & 28 & 36 & 42 & 38 & 18 & 20 & $4-7$ & 40 & 40 & 38 & 42 & 30 & 80 & 14 & 36 & 60 & \\
\hline & $10-$ & & & & & & $15-$ & $15-$ & $10-$ & $25-$ & $20-$ & & $15-$ & $30-$ & $30-$ & $30-$ & $25-$ & $10-$ & $10-$ & \\
\hline Shrub layer coverage $(\%)$ & 30 & 25 & 35 & 45 & 30 & 55 & 75 & 40 & 55 & 65 & 45 & 35 & 45 & 60 & 75 & 80 & 75 & 15 & 30 & \\
\hline Herbaceous layer coverage & & $60-$ & $45-$ & & $60-$ & $70-$ & $60-$ & & $35-$ & & $60-$ & & & & & & & & & \\
\hline$(\%)$ & 100 & 85 & 90 & 100 & 75 & 90 & 85 & 100 & 90 & 100 & 85 & 100 & 100 & 90 & 100 & 100 & 90 & 90 & 100 & \\
\hline Number of species & 58 & 28 & 20 & 23 & 35 & 34 & 36 & 28 & 26 & 27 & 45 & 22 & 27 & 17 & 25 & 18 & 26 & 31 & 62 & \\
\hline \multicolumn{21}{|l|}{$\begin{array}{l}\text { Characteristic species } \\
\text { ass. Salicetum albae }\end{array}$} \\
\hline Salix alba & $1-3$ & 1 & 1 & 4 & 3 & 2 & $1-3$ & 4 & $3-4$ & 4 & 3 & 1 & 4 & + & 3 & $4-5$ & 1 & 4 & 1 & V \\
\hline Cornus sanguinea & 2 & 2 & - & - & $1-2$ & 1 & $1-4$ & 2 & $1-3$ & $2-3$ & 2 & 1 & 1 & $2-3$ & $2-4$ & 1 & 4 & 2 & 2 & V \\
\hline Rubus caesius & $3-5$ & $2-4$ & $3-4$ & $2-4$ & $2-3$ & $2-3$ & $2-5$ & $3-5$ & $2-3$ & $2-4$ & 3 & $3-4$ & $2-4$ & $2-3$ & 1 & $3-4$ & $2-3$ & $3-4$ & $3-4$ & V \\
\hline Glechoma hederacea & 2 & 1 & 1 & $1-2$ & 1 & 3 & $1-2$ & 1 & $1-2$ & $3-4$ & 3 & $1-2$ & $1-2$ & $1-2$ & 3 & - & 1 & 2 & $1-2$ & V \\
\hline Urtica dioica & 2 & 1 & - & 2 & 2 & $2-3$ & $2-3$ & 2 & 2 & 3 & 1 & 2 & 1 & $1-4$ & $2-5$ & 1 & - & - & $3-5$ & IV \\
\hline Salix fragilis & 1 & 1 & - & - & 1 & 1 & 1 & - & - & - & 1 & - & - & 1 & - & - & - & 1 & 1 & III \\
\hline $\begin{array}{l}\text { Vitis sylvestris } \\
\text { Char.subass. }\end{array}$ & - & - & - & - & + & + & - & - & - & - & - & - & + & - & + & - & 1 & $1-2$ & + & III \\
\hline Leucojum aestivum & $1-3$ & $1-3$ & + & $1-2$ & $1-2$ & + & + & $1-3$ & $1-3$ & 1 & $1-2$ & $1-2$ & + & $3-4$ & 1 & 1 & $1-3$ & $1-2$ & 1 & $\overline{\mathrm{V}}$ \\
\hline Iris pseudacorus & + & + & + & 1 & + & + & + & + & $1-2$ & + & 1 & + & 1 & - & + & + & 1 & 1 & + & $\mathrm{V}$ \\
\hline Carex riparia & - & - & - & $1-2$ & - & 2 & 2 & $2-3$ & 2 & - & 2 & 2 & 2 & - & 2 & - & 3 & 3 & 2 & IV \\
\hline Ficaria verna & $2-3$ & 2 & $1-4$ & 2 & 1 & $1-2$ & $2-3$ & 1 & 2 & $3-4$ & 3 & 2 & - & 2 & 1 & 1 & - & - & - & IV \\
\hline Diff. facies & & & & & & & & & & & & & & & & & & & & \\
\hline $\begin{array}{l}\text { Populus alba } \\
\text { Salicion albae }\end{array}$ & - & 1 & 4 & - & 1 & $2-3$ & $3-4$ & + & + & 1 & 2 & 1 & - & 4 & $1-2$ & $1-2$ & 4 & - & 4 & IV \\
\hline Acer negundo & 1 & 1 & 1 & $1-3$ & 1 & 3 & 1 & 1 & - & $1-2$ & 2 & + & + & 1 & 1 & - & 1 & - & 3 & V \\
\hline Valeriana officinalis & + & + & + & + & + & - & + & $1-2$ & - & - & + & - & - & - & - & - & - & - & - & III \\
\hline Viburnum opulus & - & + & - & - & + & - & - & - & - & - & - & - & - & - & - & - & + & + & + & II \\
\hline Humulus lupulus & 1 & - & 1 & - & + & - & - & - & - & $1-2$ & 1 & - & - & - & + & - & 1 & - & - & II \\
\hline Phalaris arundinacea & - & - & - & - & - & - & - & - & - & - & I & - & 2 & - & - & - & 1 & - & - & II \\
\hline
\end{tabular}


PAVEL PÎNZARU

\begin{tabular}{|c|c|c|c|c|c|c|c|c|c|c|c|c|c|c|c|c|c|c|c|c|}
\hline Amorpha fruticosa & + & - & - & - & - & - & - & - & 1 & 2 & - & - & $1-2$ & - & - & - & 1 & - & - & II \\
\hline Frangula alnus & - & - & - & - & - & - & - & - & - & - & - & - & - & - & - & - & - & $\mathrm{r}$ & - & I \\
\hline Viola elatior & - & - & - & - & - & - & - & - & + & - & - & - & - & - & + & - & - & - & + & I \\
\hline Galium rubioides & 2 & - & - & - & - & - & - & - & - & - & - & - & - & - & - & 2 & - & - & 2 & I \\
\hline Asparagus pseudoscaber & $\mathrm{r}$ & - & - & - & $\mathrm{r}$ & - & - & - & - & - & + & - & - & - & - & - & - & $\mathrm{r}$ & - & I \\
\hline $\begin{array}{l}\text { Silene bacifera } \\
\text { Salicion triandrae }\end{array}$ & - & - & - & - & - & - & - & - & - & - & + & - & - & - & - & - & - & - & - & I \\
\hline Galium aparine & 2 & 1 & 1 & 1 & 1 & $3-4$ & $1-2$ & 1 & $1-2$ & 2 & 2 & $1-2$ & - & 2 & 3 & $2-3$ & 1 & - & 1 & V \\
\hline Lysimachia vulgaris & - & - & - & + & + & - & - & - & - & - & + & - & + & - & - & - & - & + & - & II \\
\hline Calystegia sepium & + & - & - & - & - & - & - & - & - & - & + & - & - & - & - & - & - & - & - & I \\
\hline $\begin{array}{l}\text { Rumex obtusifolium } \\
\frac{\text { Salicetalia et Salicetea }}{\text { purpureae }}\end{array}$ & + & - & - & - & + & - & + & - & - & - & - & - & - & - & - & - & - & - & + & I \\
\hline Symphytum officinalis & + & 1 & + & + & + & + & + & + & + & + & + & - & + & - & + & + & 1 & + & + & $\mathrm{V}$ \\
\hline Lysimachia nummularia & 2 & - & - & - & 2 & 2 & 2 & $2-3$ & 2 & - & 2 & $2-3$ & 2 & 2 & $2-3$ & - & 2 & 3 & 2 & IV \\
\hline Poa palustris & 2 & - & - & - & $1-2$ & $1-2$ & - & 3 & - & - & 1 & 3 & 1 & - & - & - & - & - & 1 & III \\
\hline Populus nigra & $1-3$ & + & - & - & $1-2$ & 1 & - & - & 2 & - & 1 & - & $1-2$ & - & - & - & - & - & - & II \\
\hline Solanum dulcamara & + & - & - & - & - & - & - & - & - & - & + & - & - & - & - & - & - & - & - & I \\
\hline Salix purpurea & - & 1 & - & - & - & - & 1 & - & - & - & - & - & - & - & - & - & - & - & - & I \\
\hline $\begin{array}{l}\text { Rumex sanguineus } \\
\text { Phragmitetea s.l. }\end{array}$ & - & 1 & - & + & + & + & - & - & - & - & - & - & - & - & - & - & - & - & - & I \\
\hline Phragmites australis & - & - & - & - & - & - & 2 & - & - & 3 & - & - & - & - & 1 & - & - & - & 1 & II \\
\hline Carex melanostachya & - & - & - & - & - & - & - & - & - & - & - & - & - & - & $1-2$ & - & - & - & - & I \\
\hline Epilobium ciliatum & - & - & - & - & - & - & - & - & - & - & - & - & - & - & + & - & - & - & - & I \\
\hline Inula helenium & - & - & - & - & - & - & - & - & - & - & - & - & - & - & - & - & - & $\mathrm{r}$ & - & I \\
\hline Eupatorium cannabinum & - & - & - & - & - & - & - & - & - & - & - & - & - & - & - & - & - & - & 1 & I \\
\hline Rorippa austriaca & - & - & - & - & - & - & - & - & - & - & - & - & - & - & - & - & - & - & + & I \\
\hline $\begin{array}{l}\text { Alisma plantago-aquatica } \\
\text { Molinio-Arrhenatheretea s.l. }\end{array}$ & + & - & - & - & + & - & + & - & - & - & - & - & - & - & - & - & - & & + & I \\
\hline Taraxacum camylodes & + & - & - & - & - & + & + & + & + & + & - & + & - & - & + & + & - & - & - & III \\
\hline Euphorbia lucida & + & - & + & - & + & - & + & - & - & + & + & - & 1 & - & + & - & - & + & + & III \\
\hline Phleum pratense & - & - & - & - & - & + & - & - & 1 & 2 & - & - & + & - & - & - & - & - & 1 & II \\
\hline Potentilla reptans & - & - & - & - & - & - & - & - & 1 & + & - & - & $1-2$ & - & - & - & - & - & 2 & II \\
\hline Carex hirta & - & - & - & - & - & - & - & - & - & - & - & - & - & - & 3 & 3 & - & - & $1-2$ & I \\
\hline Daucus carota & + & - & - & - & - & - & - & + & - & - & - & - & + & - & - & - & - & - & - & I \\
\hline Prunella vulgaris & + & - & - & - & - & - & - & - & - & - & - & - & 1 & - & - & - & - & - & + & I \\
\hline Rorippa sylvestris & - & - & - & - & - & - & - & - & - & - & - & - & - & - & - & - & - & - & + & I \\
\hline
\end{tabular}


SALICETUM ALBAE ISSLER 1924 LEUCOJETOSUM AESTIVI PÎNZARU SUBASS. NOV. IN...

\begin{tabular}{|c|c|c|c|c|c|c|c|c|c|c|c|c|c|c|c|c|c|c|c|c|}
\hline Scutellaria hastifolia & - & - & - & - & - & - & - & - & - & - & - & - & - & - & - & - & - & 1 & - & I \\
\hline Thalictrum lucidum & - & - & - & - & - & - & - & - & - & - & - & - & - & - & - & - & - & - & 1 & I \\
\hline Vicia sepium & - & + & - & - & - & - & - & - & - & - & - & - & - & - & - & - & - & - & - & I \\
\hline Trifolium repens & - & 1 & - & - & - & - & - & - & - & - & + & - & - & - & - & - & - & - & - & I \\
\hline Cerastium holosteoides & - & + & - & - & - & - & - & - & - & - & + & - & - & - & - & - & - & - & - & I \\
\hline Equisetum arvense & - & - & - & - & - & - & - & - & - & - & 1 & - & - & - & - & - & - & 2 & - & I \\
\hline Ranunculus polyanthemos & - & - & - & - & - & - & - & - & - & - & - & - & - & - & - & - & - & - & + & I \\
\hline Trifolium campestre & - & - & - & - & - & - & - & - & - & - & - & - & - & - & - & - & - & - & + & I \\
\hline $\begin{array}{l}\text { Trifolium pratense } \\
\text { Querco-Fagetea s.l. }\end{array}$ & - & - & - & - & - & - & - & - & - & - & - & - & - & - & - & - & - & - & + & I \\
\hline Fraxinus excelsior & - & 3 & 1 & 1 & 2 & - & - & - & - & - & 1 & 3 & - & 1 & 1 & 1 & - & 1 & + & IV \\
\hline Quercus robur & $1-2$ & - & - & - & - & - & - & - & - & - & - & - & - & + & - & - & 1 & 1 & 1 & II \\
\hline Chaerophyllum temulum & + & - & - & - & + & - & - & - & - & - & + & - & - & + & - & - & 1 & - & 1 & II \\
\hline Malus sylvestris & - & - & - & - & - & - & - & + & + & - & - & - & - & - & - & - & - & - & - & I \\
\hline Cephalanthera damasonium & - & - & - & - & - & - & - & - & - & - & - & - & - & - & - & - & - & - & $\mathrm{r}$ & I \\
\hline Epipactis helleborine & - & - & - & - & - & - & - & - & - & - & - & - & - & - & - & - & $\mathrm{r}$ & - & $\mathrm{r}$ & I \\
\hline Convallaria majalis & 2 & - & - & - & - & - & - & - & - & - & - & - & - & - & - & - & - & 2 & 2 & I \\
\hline Carex contigua & - & - & - & - & - & - & - & - & - & - & - & - & - & - & - & - & - & 1 & 1 & I \\
\hline Ulmus minor & - & - & 1 & - & - & $1-2$ & $1-2$ & - & - & - & - & - & - & - & - & - & - & - & 1 & I \\
\hline Ulmus glabra & - & - & - & - & - & - & - & - & - & - & - & - & - & - & - & - & - & - & 1 & I \\
\hline Euonymus europaeus & + & - & + & - & - & - & - & - & - & - & - & - & - & - & - & - & - & - & - & I \\
\hline Geum urbanum & 1 & + & + & - & - & + & - & - & - & - & + & - & - & - & - & - & - & - & - & I \\
\hline Lapsana communis & + & - & - & - & - & - & - & - & - & - & + & - & - & - & - & - & - & - & + & I \\
\hline Salix cinerea & & - & - & + & - & - & - & - & - & - & - & - & - & - & - & - & - & - & - & I \\
\hline Populus tremula & - & - & - & - & - & - & - & - & - & - & + & - & - & - & - & - & - & - & & I \\
\hline $\begin{array}{l}\text { Polygonatum latifolium } \\
\text { Rhamno-Prunetea s.l. }\end{array}$ & - & - & - & - & - & - & - & - & - & - & - & - & - & - & - & - & 1 & - & & I \\
\hline Crataegus monogyna & 2 & 2 & 2 & + & 1 & 1 & 1 & $1-2$ & - & + & 1 & + & - & 1 & + & 1 & + & + & 1 & $\mathrm{~V}$ \\
\hline Prunus spinosa & + & - & - & - & + & 1 & $1-3$ & 1 & 1 & $1-3$ & + & 1 & - & - & - & - & - & - & 1 & III \\
\hline Pyrus pyraster & + & + & - & + & + & + & 1 & + & + & + & + & - & + & - & - & - & - & - & - & III \\
\hline Corylus avellana & - & - & - & - & - & - & - & - & - & - & - & - & - & - & - & - & - & - & + & I \\
\hline Acer tataricum & 1 & - & $1-2$ & 1 & - & - & 1 & - & - & - & - & - & - & - & - & - & - & - & - & I \\
\hline Vicia tenuifolia & - & - & - & + & + & - & - & - & - & - & - & - & 2 & - & - & - & - & - & $1-2$ & I \\
\hline Rosa canina & + & + & - & - & + & - & - & - & - & - & - & - & - & - & - & - & - & - & - & I \\
\hline $\begin{array}{r}\text { Ligustrum vulgare } \\
\underline{\text { Aliae }}\end{array}$ & & - & - & - & - & - & - & - & - & - & - & - & - & - & - & - & 1 & - & - & I \\
\hline Stellaria media & 1 & $1-2$ & 1 & 1 & 1 & $2-3$ & $1-2$ & $1-2$ & $2-3$ & $2-3$ & 2 & $1-2$ & - & 2 & - & 2 & - & - & - & IV \\
\hline
\end{tabular}


PAVEL PÎNZARU

\begin{tabular}{|c|c|c|c|c|c|c|c|c|c|c|c|c|c|c|c|c|c|c|c|c|}
\hline Arctium tomentosum & + & + & - & - & + & + & - & + & + & - & + & + & - & + & + & - & + & - & + & IV \\
\hline Elymus repens & 2 & $2-3$ & 2 & $3-4$ & $3-4$ & - & $3-4$ & 3 & 4 & - & - & - & $2-3$ & - & - & - & - & 3 & $3-4$ & IV \\
\hline Morus nigra & + & - & - & - & - & - & 1 & - & - & + & + & + & + & - & + & - & + & + & + & III \\
\hline Lamium purpureum & 2 & - & - & - & - & 1 & + & 1 & $1-3$ & $2-3$ & + & - & - & - & $1-2$ & + & - & - & - & III \\
\hline Anthriscus longirostris & $1-3$ & - & - & - & 3 & $2-3$ & 2 & 1 & - & $1-2$ & + & - & - & - & 2 & - & - & - & 2 & III \\
\hline Cirsium arvense & + & - & - & - & - & + & + & + & - & - & + & - & - & - & - & + & - & - & + & II \\
\hline Aristolochia clematitis & 2 & - & - & - & 1 & - & 2 & 2 & - & - & - & - & - & - & - & - & 2 & 2 & 2 & II \\
\hline Euphorbia virgata & + & + & - & - & - & - & + & - & + & - & - & - & + & - & - & - & - & - & - & II \\
\hline Valerianella carinata & - & - & - & - & - & + & + & 1 & + & - & - & - & - & - & - & - & - & - & - & II \\
\hline Lathyrus tuberosus & - & - & + & + & - & - & - & - & - & - & - & - & - & - & - & - & - & 1 & 1 & II \\
\hline Vicia hirsuta & + & - & - & - & - & - & 1 & - & - & - & - & - & 1 & - & - & - & - & + & - & II \\
\hline Gleditsia triacanthos & + & - & - & - & - & - & - & - & - & - & + & - & - & - & - & - & - & + & - & I \\
\hline Alliaria petiolata & - & - & - & - & - & - & + & + & - & 3 & - & - & - & - & - & - & - & - & - & I \\
\hline Ballota nigra & + & - & - & - & - & + & - & - & - & - & - & + & - & - & - & - & - & - & - & I \\
\hline Erigeron annuus & + & - & - & - & - & - & - & - & - & - & - & - & - & - & - & - & - & + & + & I \\
\hline Torilis arvensis & - & - & - & - & + & - & - & - & - & - & - & - & - & - & - & - & - & - & + & I \\
\hline Robinia pseudacacia & + & - & - & - & - & - & - & - & - & - & - & - & - & - & - & - & - & 1 & - & I \\
\hline Morus alba & - & - & - & - & - & - & - & - & - & - & - & - & - & - & - & - & - & - & + & I \\
\hline Fraxinus americana & + & - & - & - & - & - & - & - & - & - & - & - & - & - & - & - & - & 1 & 1 & I \\
\hline Artemisia absinthium & - & - & - & - & - & - & - & - & - & - & - & - & - & - & - & - & - & - & + & I \\
\hline Leonurus cardiaca & - & - & - & + & - & + & - & - & - & - & - & - & - & - & - & - & - & - & - & I \\
\hline Tanacetum vulgare & + & - & - & - & - & - & - & - & - & - & - & - & - & - & - & - & - & - & - & I \\
\hline Plantago major & + & - & - & - & - & - & - & - & - & - & + & - & - & - & - & - & - & - & - & I \\
\hline Cardaria draba & - & - & - & - & - & - & 1 & - & - & - & - & - & - & - & - & - & - & - & - & I \\
\hline Verbascum blattaria & - & - & - & - & - & - & - & - & - & - & - & - & - & - & - & - & - & - & $\mathrm{r}$ & I \\
\hline Lathyrus sylvestris & - & - & - & - & - & - & - & - & - & - & - & - & - & - & - & - & - & - & + & I \\
\hline Calamagrostis epigeios & - & - & - & - & - & - & - & - & - & - & - & - & 2 & - & - & - & - & - & - & I \\
\hline Odontites vulgaris & - & + & - & - & - & - & - & - & - & - & - & - & - & - & - & - & - & - & - & I \\
\hline Vicia tetrasperma & - & - & - & + & - & - & - & - & + & - & - & - & - & - & - & + & - & - & - & I \\
\hline Lamium maculatum & - & - & - & - & 1 & - & - & - & - & - & 1 & - & - & - & - & - & - & - & - & I \\
\hline Equisetum ramosissimum & - & - & - & - & - & - & - & - & - & - & - & - & - & - & - & - & - & 2 & - & I \\
\hline Plantago lanceolata & - & - & - & - & - & - & - & - & - & - & - & - & - & - & - & - & - & - & + & I \\
\hline
\end{tabular}

Place and date of the relevés: rel. 1-2, Călmăuți commune, Hîncești district, 22.IV.2018, 12.V.2018, 22.VIII.2018; rel. 3-6, Dancu commune, Hîncești district, 22.IV.2018, 22.VIII.2018; rel. 7, 8, *9 (typus), 10, Cioara commune, Hîncești district, 22.IV.2018; 22.VIII.2018; rel. 11 Leușeni commune, Hîncești district, 19.VI.2018; rel. 12-15, SărataRăzesi commune, Leova district, 24.IV.2018, 13.V.2018; rel. 16-17, Cotul Morii commune, Hîncesti district, 19.VI.2018; rel. 18-19, Nemteni commune, Hîncești district, 10.VI.2018. facies populosum albae: rel. 3, 6, 7, 14, 19. 


\section{References}

AESCHIMANN D., LAUBER K., MOSER D. M. \& THEURILLAT J. P. 2004. Flora alpina. Bologna: Zanichelli.Vol. 2, $1188 \mathrm{pp}$.

BRAUN-BLANQUET J. 1964. Pflanzensoziologie. Grundzüge der Vegetationskunde. Ed. 3. Wien: Springer Verlag, 865 pp.

CANTEMIR V. \& ALEXANDROV E. 2015. Vitis sylvestris C.C.Gmel. Pp. 114. In: Cartea Roşie a Republicii Moldova The Red Book of the Republic of Moldova. Col. red. acad. Gh. DUCA. Edit. 3, 2015. Chișinău: Știința, 492 pp.

CHIFU T. \& IRIMIA I. 2014. Salicetea purpureae Moor 1958. Pp. 7-44. In: CHIFU Th. (eds) Diversitatea fitosociologică a vegetației României. Part. III. Vegetația pădurilor și tufărișurilor. Iași: Institutul European, 551 pp.

CHIFU T. \& MITITELU D. 1992. Vegetația Moldovei. Vegetația de luncă. Pp 77-81. In: DONIȚĂ N., IVAN D., COLDEA G., SANDA V., POPESCU A., CHIFU Th., PAUCĂ-COMANESCU M., MITITELU D. \& BOȘCAIU N. 1992. Vegetația României. București: Edit. Tehnică Agricolă, 407 pp.

COLDEA Gh. 2015. Ass. Salicetum albae Issler 1926. Pp. 15. Tab. synt. 1, col. 2a, 2b. In: COLDEA Gh. (eds) Les associations végétales de Roumanie. Tome 3. Les associations forestiéres et arbustives. Cluj-Napoca: Presa Universitară Clujeană, 281 pp.

COVALI V. 2008. Floristic and phytocoenotic biodiversity of protected area Nemțeni (R. Moldova). J. Plant Develop. 15: 69-76.

ELLENBERG H, WEBER H. E., DÜLL R., WIRTH V., WERNER W. \& PAULIßEN D. 1992. Zeigerwerte von Pflanzen in Mitteleuropa. Göttingen: Erich Goltze KG, 258 pp.

GAFTA D., NICULESCU M., OPREA A., SÂRBU I., COLDEA G. \& ALEXIU V. 2008. Păduri temperate europene. 91E0 Păduri aluviale. Pp.70. In: Manual de intrepetare a habitatelor Natura 2000 din Românial Dan GAFTA \& Owen MOUNTFORD (coord.). - Cluj-Napoca: Risoprint.101 pp.

GHEIDEMAN T. S., OSTAPENKO B. F., NICOLAEVA L. P., ULANOVSKIJ M. S. \& DMITRIEVA N. V. 1964. Tipy lesa i lesnye assotziatzii Moldavskoj SSR. Kišinev: Cartea moldovenească, 268 pp.

GHENDOV V. \& CIOCÂRLAN N. 2015. Leucojum aestivum L. Pp. 124. In: Cartea Roșie a Republicii Moldova-The Red Book of the Republic of Moldova. Col. red. acad. Gh. DUCA. Ed. 3, 2015, Chișinău: Știința, 492 pp.

IVAN D., DONIȚĂ N., COLDEA Gh., SANDA V., POPESCU A., CHIFU Th., BOȘCAIU N. MITITELU D. \& PAUCĂ-COMANESCU M. 1993. Végétation potentielle de la Roumanie. Braun-Blanquetia. Recueil de travaux de geobotanique reliew of geobotanical monographs. Camerino. 9: 79.

NEDEALCOV M., RĂIILEAN V., CHIRICĂ L. \& al. 2013. Atlas. Resursele climatice ale Republicii Moldova = Atlas . Climatic resources of the Republic of Moldova. Chișinău: Î. E. P. Ştiinţa, 76 pp.

NEUHÄUSLOVÁ Z. \& DOUDA J. 2013: KAC01 Salicetum albae Issler 1926. Pp. 64-66. In: CHYTRÝ M. (ed.), Vegetace České republiky. 4. Lesni a krovinná vegetace [Vegetation of the Czech Republic 4. Forest and shrub vegetation]. Praha: Academia. $552 \mathrm{~s}$.

PÎNZARU P. \& SÎRBU T. 2016. Flora vasculară din Republica Moldova. (Lista speciilor și ecologia). Chișinău: Tipografia UST, $261 \mathrm{pp}$.

PÎNZARU P. Ia. 1991. Srednepridnestrodskie lesa Moldovy (fitotzenologhia, tipologhia, flora, ohrana) 03.00.05 botanica. Avtoreferat diss. kand. nauk. Kišinev, 25 pp.

POSTOLACHE G. 1995. Vegetația Republicii Moldova. Chișinău: Știința, 340 pp.

POSTOLACHE G. 2017. Rezervația „Pădurea Domnească”. Chișinău: Combinatul Poligrafic, 256 pp.

POSTOLACHE G. \& CHIRTOACĂ V. 2005. Vegetația. Pp.167-223. In: Natura rezervației „Plaiul Fagului”. Sub red. acad. A.URSU. Chișinău-Rădenii Vechi, 431 pp.

POSTOLACHE G. \& JARDAN N. Cephalanthera damasonium (Mill.) Druce. Pp. 152. In: Cartea Roșie a Republicii Moldova - The Red Book of the Republic of Moldova. Col. red. acad. Gh. DUCA. Ed. 3, 2015, Chișinău: Știința, 492 pp.

POSTOLACHE G. \& POSTOLACHE D. 2012.Vegetația. Pp. 50-86. In: POSTOLACHE Gh, MUNTEANU A., POSTOLACHE D. \& COJAN C. Rezervația "Prutul de Jos". Chișinău: Tipografia Centrală, 152 pp.

SÂRBU I., ȘTEFAN N. \& OPREA A. 2013. Plante vasculare din România. Determinator ilustrat de teren. București: Edit. Victor B Victor, 627 pp.

SEIBER P. \& CONARD M. 1992: Ass. Salicetum albae Issler 1926. A. Textbland, Pp. 22-23. B. Tabellenband, Tabelle 241. Assoziation Nr. 6, Pp.11-12. In: OBERDORFER E. (eds) Süddeutsche Pflanzengesellschaften . Teil IV. Wälder und Gebüsche. Gustav Fischer Verlag, Jena. A. Textband, 282 pp. B. Tabellenband, 580 pp.

SÍLC U. 2003. Vegetation of the class Salicetea purpureae in Dolenjska (SE Slovenia). Fitosociologia. 40(2): 3-27.

TOFAN-BURAC T. \& CHIFU T. 2002. Flora şi vegetația din valea Prutului. Iași: Edit. CORSON, 437 p.

${ }^{* * *}$ Legea privind fondul ariilor naturale protejate de stat $\mathrm{Nr} .1538$ din 25.02.1998. Monitorul oficial din 16.07.1998, Nr. 066 art. Nr. 442.

How to cite this article:

PÎNZARU P. 2018. Salicetum albae Issler 1924 leucojetosum aestivi Pînzaru subass. nov. in the Republic of Moldova. J. Plant Develop. 25: 145-164. https://doi.org/10.33628/jpd.2018.25.1.145 